\title{
Evaluación Económica de la Vacuna contra la Influenza aplicada al Personal de Salud que Atiende Pacientes Oncológicos Hospitalizados
}

\author{
Economic evaluation of influenza vaccine applied to health \\ personnel attending hospitalised oncological patients
}

\author{
Liliana A. Chicaíza-Becerra ${ }^{1}$, Mario García-Molina¹, Mónica Ballesteros², Oscar \\ Gamboa $^{2}$, Jorge Díaz ${ }^{3}$ y Ricardo Vega ${ }^{4}$
}

\author{
1 Facultad de Ciencias Económicas, Universidad Nacional de Colombia.lachicaizab@unal.edu.co, \\ mgarciamo@unal.edu.co \\ 2 Médica. Especialista en epidemiología. Grupo de Investigación Clínica, Instituto Colombiano de \\ Cancerología.mpballesteross@unal.edu.co,ogamboag@unal.edu.co \\ 3 Facultad de Ciencias, Universidad Nacional de Colombia. jadiazr@unal.edu.co \\ 4 Investigador independiente. ravegaro@unal.edu.co
}

Recibido 9 Noviembre 2007/Enviado para Modificación 3 Octubre 2008/Aceptado 3 Noviembre 2008

\section{RESUMEN}

Objetivos Analizar, desde la perspectiva del pagador, el costo efectividad de la vacunación contra influenza al personal de salud que tiene contacto estrecho con los pacientes oncológicos hospitalizados en Colombia.

Métodos Mediante un árbol de decisión, se identificó la relación de costo-efectividad de la aplicación de esta vacuna al personal de salud que tiene contacto estrecho con los pacientes oncológicos hospitalizados. La perspectiva es la del pagador. Los costos se valoraron en unidades monetarias y la efectividad se mide por la reducción en días de hospitalización de pacientes oncológicos como resultado de la disminución en las probabilidades de contraer el virus por la vacunación al personal de salud.

Resultados Vacunar contra la influenza al personal de salud que tiene contacto estrecho con los pacientes oncológicos hace que, al ahorrar un día de estancia hospitalaria, simultáneamente se ahorren \$ 2978000 (US\$ 1 324).

Conclusiones Vacunar contra la influenza al personal de salud que tiene contacto estrecho con los pacientes oncológicos es costo efectivo para una probabilidad de contagio del personal de salud que no ha sido vacunado mayor o igual a 0,02.

Palabras Clave: Influenza humana, vacunas contra la influenza, costo efectividad, modelos económicos, vacunas, cáncer (fuente: DeCS, BIREME). 


\section{ABSTRACT}

Objective Performing a cost-effectiveness evaluation of influenza vaccine for health workers coming into close contact with oncological patients from the payer's point of view.

Methods As no evidence was found for influenza vaccine effectiveness in oncological patients, the cost-effectiveness of applying the vaccine to health workers coming into close contact with oncological patients was calculated by means of a decision tree; the payer's point of view was adopted. Costs were evaluated in monetary units and effectiveness was measured by the reduction in length of hospital stay for cancer patients as a result of lesser probability of catching the disease because of vaccinating the health workers.

Results Vaccinating health workers coming into close contact with cancer patients led to savings of $\$ 2978000$ (US\$1 324) per day of stay saved.

Conclusions Vaccinating health workers coming into close contact with cancer patients proved cost-effective, returning health worker contagion probability higher than or equal to 0.02 .

Key Words: Influenza, human influenza vaccine, cost-benefit analysis, economic model, vaccination, neoplasm (source: $\mathrm{MeSH}, \mathrm{NLM}$ ).

$\mathrm{L}$

a vacunación contra el virus de la influenza se recomienda para disminuir la morbilidad, hospitalización y mortalidad causada por las enfermedades derivadas del virus así como para evitar las complicaciones que éste genera en pacientes inmunocomprometidos. En Estados Unidos se estima que al año se producen 16000 egresos por enfermedades relacionadas con el virus de la influenza en pacientes con cáncer. La estancia hospitalaria media por esta razón es de aproximadamente 6 días y el costo medio es de 6300 dólares del año 2003. El 9 \% de los pacientes muere en el hospital y el $31 \%$ requiere rehabilitación. La tasa estimada para hospitalización en menores de 65 años es de 219 por 100000 y de muerte es de 17,4 por 100000 . Y para mayores de 65 años las tasas ascienden a 623 y 59,4 respectivamente (1).

En Colombia, no se conoce el impacto del virus de la influenza en pacientes oncológicos, no obstante, se tienen documentadas virológicamente las epidemias que han afectado a la población general en los años 1994 y $1996(2,3)$ y desde 1997 se han realizado aislamientos para detectar los tipos de virus (4-6).

Las complicaciones que genera el virus en pacientes inmunocomprometidos, que pueden ocasionarle la muerte, han llevado a proponer la aplicación de vacunas a los pacientes oncológicos (7-9). Sin embargo, la revisión sistemática de la bibliografía realizada por el Grupo de Investigación Clínica en Cáncer del 
Instituto Nacional de Cancerología para la elaboración de la guía de práctica clínica explícita basada en la evidencia, sobre la eficacia de la vacuna contra Streptococcus pneumoniae y virus influenza en pacientes oncológicos, no encontró evidencia explícita sobre la eficacia de la vacuna para este tipo de pacientes, eficacia entendida como la capacidad de la vacuna para disminuir mortalidad, evitar neumonía y enfermedades invasivas causadas por el virus de la influenza en pacientes oncológicos. La búsqueda se realizó en Embase, MEDLINE, Lilacs, CINAHL y Cochrane. En total se encontraron 352 artículos, luego de un proceso de selección realizado mediante criterios de elegibilidad por expertos temáticos y metodológicos del Instituto Nacional de Cancerología se escogieron 72 artículos. Al final sólo tres artículos (10-12) cumplieron los criterios de elegibilidad y fueron revisados de forma pareada y calificados según las plantillas de evaluación de calidad de SIGN (Scottish Intercollegiate Guidelines Network). Estos artículos no mostraron evidencia de eficacia de la vacunación en pacientes oncológicos.

No obstante, dado que existe evidencia de efectividad de la vacuna aplicada al personal de salud que se encuentra relacionado con estos pacientes (1319), se recomienda su vacunación con el fin de evitar el contagio a los pacientes oncológicos (12) que se encuentran hospitalizados.

En este artículo se presenta una evaluación de costo-efectividad de la implementación de un programa de vacunación contra la influenza al personal de salud que tiene contacto estrecho con los pacientes oncológicos hospitalizados. En Colombia no se habían realizado previamente este tipo de estudios para esta población.

\section{METODOLOGÍA}

Se utilizó un árbol de decisión para identificar las probabilidades de contagio por influenza de los pacientes oncológicos hospitalizados según esté o no vacunado el personal de salud que está en contacto estrecho con ellos. Los costos se valoran en unidades monetarias y la efectividad mediante la reducción en días de hospitalización por influenza, resultado de la implementación del programa. El análisis de costo-efectividad identifica dos escenarios, uno en el cual se vacuna al personal de salud y otro en el que no, y busca medir el efecto que tendría la reducción de probabilidades de contagio en la disminución de días de hospitalización y en consecuencia el ahorro de recursos que se lograría al implementar este programa. El modelo tiene los siguientes supuestos: 
- Identificación del número de personas del área de la salud que tienen contacto estrecho con el paciente oncológico.

Se entiende por contacto estrecho (20) cuidar o vivir con alguien o tener una alta probabilidad de contacto directo con secreciones respiratorias y/o fluidos de un paciente; en consecuencia acciones como besar o abrazar, compartir utensilios de comida o bebida, hablar con alguien en un rango de un metro de distancia y realizar el examen médico son consideradas de alto riesgo para la transmisión del virus de la influenza. En ese sentido el grupo de expertos del Instituto Nacional de Cancerología estimó que un paciente oncológico hospitalizado se relaciona en promedio con cinco personas del área de la salud (Internista, Oncólogo, Enfermera, Auxiliar de Enfermería y otro especialista).

- $\quad$ Estimación de Probabilidades

Se identificaron las probabilidades de contagio del virus de la influenza para los pacientes oncológicos hospitalizados a partir del estudio de Wilde (13). Para ambos escenarios (con vacuna y sin vacuna) es necesario identificar la probabilidad de contraer el virus y contagiarlo. El primer escenario, en el cual se vacuna al personal de salud, presenta dos situaciones: en la primera, al menos uno de los cuidadores que tiene contacto estrecho con el paciente desarrolla la infección, en este caso se supone que el paciente desarrolla la infección con una probabilidad de 1; en la segunda situación se supone que ninguno de los miembros del personal de salud contrae la infección, caso en el cual se supone que la probabilidad de infección del paciente oncológico es igual a la probabilidad de infección de la población general, debido a que los pacientes oncológicos también tienen contacto estrecho con otras personas como la familia o los amigos además del contacto que tienen con el personal de salud.

En el segundo escenario el personal de salud no se vacuna y se presentan, al igual que en el escenario anterior dos situaciones: que al menos uno se infecte, caso en el cual la probabilidad de que el paciente se infecte es de 1; o que ninguno se infecte y por lo tanto la probabilidad de que el paciente oncológico se infecte es igual a la probabilidad de infección de la población general. Para identificar estas probabilidades es necesario partir de probabilidades individuales, la Figura 1 presenta las probabilidades de que una persona del área de la salud (PAS) adquiera la infección dado que está o no vacunada, a partir de los datos de Wilde (13). 
Teniendo en cuenta, que cinco personas del área de la salud tienen contacto estrecho con el paciente oncológico (primer supuesto), es indispensable establecer las probabilidades de contagio para dicho grupo. Esto se muestra en la Figura 2.

Figura 1. Probabilidad de Contagio de una Persona del Area de la Salud (PAS)



Figura 2. Probabilidades de Contagio para el Grupo del Área de la Salud - GAS

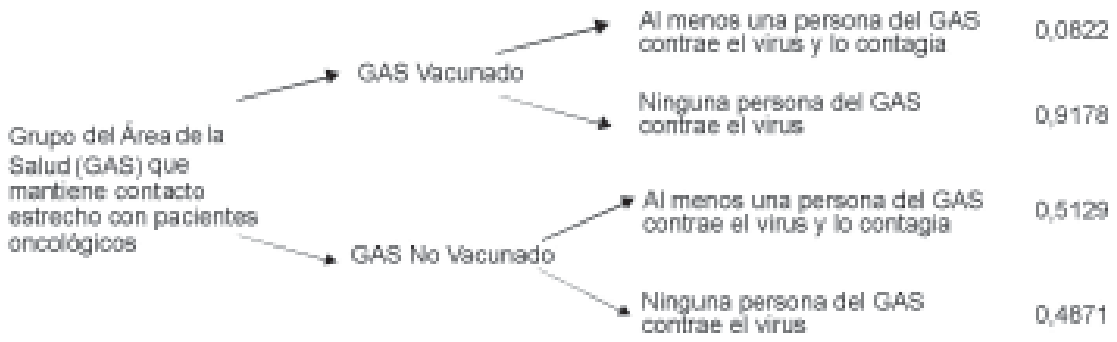

La probabilidad de que ninguno de los miembros del grupo del área de la salud-GAS, vacunados o no vacunados, adquiera el virus es igual a la probabilidad de que un PAS vacunado o no vacunado no contraiga el virus elevado a la cinco. La probabilidad de que al menos uno de los miembros del GAS adquiera la infección es 1 menos el número anterior.

Una vez se definen las probabilidades de contagio para el GAS, se identifican las probabilidades de contagio en los pacientes oncológicos con quienes el GAS tiene contacto estrecho. Esta situación se ilustra en la Figura 3. 
La probabilidad de que un paciente oncológico contraiga el virus de la influenza dado que el GAS con quien mantiene contacto estrecho está vacunado es de 0,2051 . Esta probabilidad es más del doble $(0,5782)$ cuando el GAS no esta vacunado. Estas probabilidades resultan consistentes con las estimadas por Pearson (14), quienes encontraron probabilidades de contagio que se ubican en el rango comprendido entre 25 \% y 65 \%. No obstante, se decidió sensibilizar la probabilidad de contraer el virus por parte del personal de salud que no se encuentra vacunado con el fin de identificar el efecto sobre las probabilidades finales y de esta manera establecer a partir de qué probabilidad de contagio la estrategia es costo efectiva.

Figura 3. Probabilidad de que un paciente oncológico contraiga el virus de la Influenza dado que el GAS se vacunó o no se vacunó

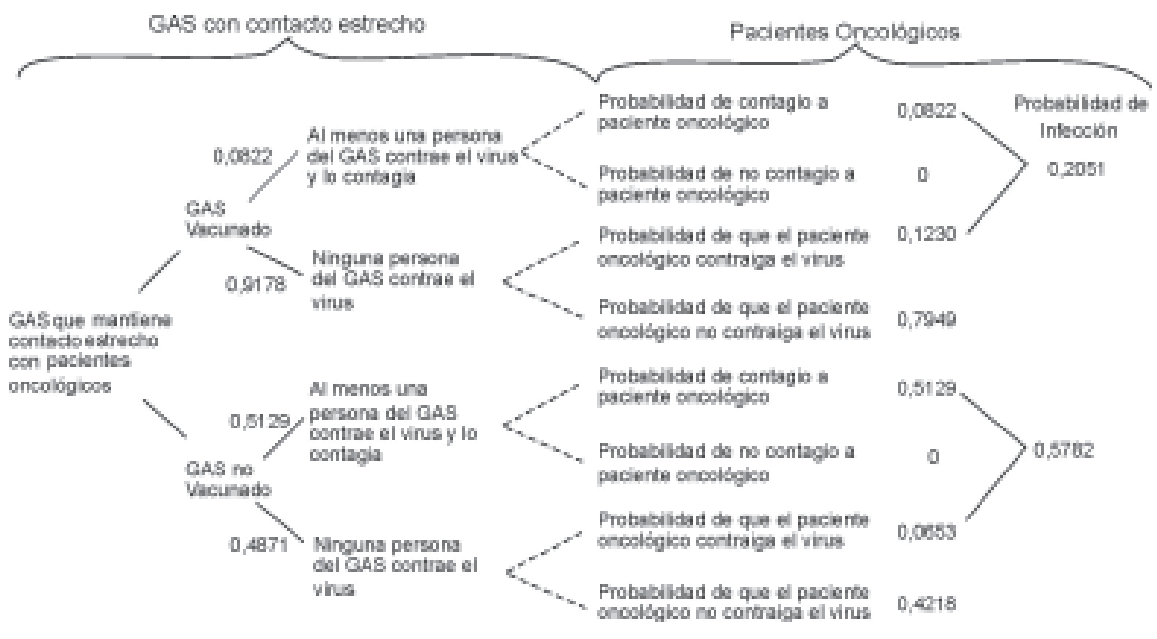

- $\quad$ Estimaciones de población

El Anuario Estadístico (21) del Instituto Nacional de Cancerología reporta para el año 2004 la atención de 6870 pacientes, de los cuales estuvieron hospitalizados 5 726. Se asume que 9500 pacientes oncológicos son hospitalizados anualmente en Colombia, con lo cual el INC tendría alrededor del $60 \%$ de esas hospitalizaciones. A partir de esta cifra se procede a aplicar las probabilidades de contagio según se vacune o no el GAS con quien mantiene contacto estrecho el paciente oncológico en el hospital. 
- $\quad$ Estimación de días de hospitalización

Los registros del Instituto Nacional de Cancerología indican que la estancia promedio de hospitalización para pacientes oncológicos es de 7,8 días, la información disponible no identifica los pacientes con complicaciones de influenza por lo tanto es necesario estimar el número de días adicionales de estancia hospitalaria cuando se presentan complicaciones por influenza. Para Estados Unidos el número de días adicionales de estancia hospitalaria es de 6 (13). El grupo de expertos del INC estimó que este dato debía ser menor para el caso colombiano y por tanto se hizo el supuesto conservador de que los días adicionales de estancia hospitalaria eran 3.

- $\quad$ Estimación de los costos

El costo diario de hospitalización se tomó a partir de las tarifas definidas por el manual único de cuentas para Colombia CUPS (22) y se estimó en \$3000 000 promedio por día de hospitalización del paciente oncológico que ha contraído el virus. También se estimó que el número total del personal de salud a ser vacunado sería de 10 000. Dado que la vacuna contra la influenza tiene un costo de \$23 200 a precios de 2007, el costo total es de \$232 000 000. Como la vacuna se aplica al personal de salud, se considera que los costos logísticos son despreciables.

Los anteriores supuestos se discutieron con un grupo de expertos en el área que incluyó a médicos especialistas en oncología clínica, infectología, epidemiología clínica y neumología oncológica con el fin de utilizar parámetros adecuados para la evaluación.

\section{RESULTADOS}

La introducción de un programa de vacunación contra el virus de la influenza al personal de salud genera un indicador de costo-efectividad \$2 978000 pesos (US\$ 1324 ), esto significa que por cada día de estancia hospitalaria evitado simultáneamente se genera un ahorro por esa cantidad. Y por lo tanto la estrategia de vacunar al personal de salud es dominante.

Al realizar la sensibilización para la variable para la cual no existe evidencia, que es la probabilidad de contagio del personal de salud no vacunado, y manteniendo los supuestos de costos y días de hospitalización, se generan los resultados de la Tabla 1. 
Tabla 1. Resultados y sensibilidad para diferentes tipos de probabilidad de contagio

\begin{tabular}{|c|c|c|c|c|}
\hline $\begin{array}{l}\text { Probabilidad } \\
\text { sensibilizada (PAS no } \\
\text { vacunada se contagie) }\end{array}$ & 0,13 & 0,08 & 0,02 & 0,01 \\
\hline $\begin{array}{l}\text { P contraer el } \\
\text { virus/GAS Vacunado }\end{array}$ & 0,21 & 0,16 & 0,10 & 0,09 \\
\hline $\begin{array}{l}\text { P contraer el virus / } \\
\text { GAS No Vacunado }\end{array}$ & 0,58 & 0,39 & 0,11 & 0,06 \\
\hline $\begin{array}{l}\text { Contagiado dado que } \\
\text { GAS se vacuna }\end{array}$ & 1949 & 1478 & 955 & 868 \\
\hline $\begin{array}{l}\text { Contagiados dado que } \\
\text { GAS no se vacuna }\end{array}$ & 5493 & 3740 & 1084 & 556 \\
\hline Costo de Vacunar \$ & 232000 & 232000 & 232000 & 232000 \\
\hline $\begin{array}{l}\text { Costos de } \\
\text { hospitalización } \\
\text { evitados cuando GAS } \\
\text { se vacuna } \$\end{array}$ & -31663758 & -20122043 & -934398 & 3037 \\
\hline $\begin{array}{l}\text { Dias de } \\
\text { hospitalización } \\
\text { evitados }\end{array}$ & -10632 & -6785 & -389 & 935 \\
\hline $\begin{array}{l}\text { Indice de Costo - } \\
\text { Efectividad \$ }\end{array}$ & 2978 & 2966 & 2403 & 3248 \\
\hline
\end{tabular}

\section{DISCUSIÓN}

En este estudio se están contabilizando los efectos directos que tendría la aplicación de la vacuna a los pacientes oncológicos; sin embargo, cabe anotar que los beneficios indirectos no se han contemplado. El más significativo tiene que ver con las externalidades derivadas de la aplicación de la vacuna al personal de salud que trabaja en diferentes tipos de hospitales y cuida a diferentes tipos de pacientes, quienes se verán afectados positivamente por la introducción de esta medida, sin mencionar la disminución en las molestias derivadas de la adquisición de la enfermedad. La decisión de implementar un programa de salud de este estilo resulta costo-efectiva si sólo se considera el beneficio sobre pacientes oncológicos y por lo tanto el beneficio es aún mayor si se considera a la población total.

Dado que la vacunación genera externalidades positivas importantes, ya que no sólo se beneficia el personal vacunado sino también otros individuos, el costo de la vacuna no debería recaer sobre el personal de salud. En ese sentido se recomienda que los costos del programa de vacunación sean asumidos por los aseguradores públicos y privados o por el Estado, cuyos costos se reducirían. 
En la literatura no se encontraron artículos que evaluaran la costo-efectividad de vacunar al personal de salud que atiende pacientes oncológicos. No obstante se identificó el estudio de Avritscher (19), el cual evalúa la costo-efectividad de la vacuna contra la influenza en pacientes oncológicos, encontrando que la intervención es costo-efectiva (RCEI US \$ 224 por QALY). Sin embargo este estudio asume que la vacuna es efectiva para títulos de anticuerpos inhibidores de la hemaglutinación (AIH) $\geq 40$, títulos que son protectores para personas sanas pero no está claro que lo sean en pacientes oncológicos (19). El estudio de Kempe encontró que el $24 \%$ de los pacientes con cáncer con $\mathrm{AIH} \geq 32$ desarrollaron influenza comparado con ningún caso en las personas sin cáncer que tenían el mismo nivel de AIH (23). Dado que no hay evidencia de la efectividad de la vacuna en pacientes oncológicos y no se conocen los títulos de anticuerpos que son protectores en este tipo de pacientes, los supuestos de efectividad son fuertes y pueden conducir los resultados de la evaluación.

El presente estudio tiene varias limitaciones como todos los estudios de evaluación económica en los cuales se utilizan modelos y supuestos. El principal supuesto fue la tasa de transmisión de la infección desde el personal de salud a los pacientes con cáncer; a pesar de ser un supuesto fuerte en los análisis de sensibilidad se encuentra que la intervención es costo efectiva incluso con probabilidades de contagio del 0,01. El presente estudio no incluyó los costos de los eventos adversos a la vacunación lo cual podría sobreestimar la costo efectividad de la vacuna. Sin embargo en la literatura se informa que la mayoría de eventos adversos son reacciones locales y eventos sistémicos leves y transitorios (24-26). Los costos del tratamiento de un caso de influenza no fueron incluidos en el análisis esto podría subestimar la costo-efectividad de la vacunación, pese a esto la intervención es altamente costo-efectiva.

Se deben realizar estudios clínicos aleatorizados que evalúen la eficacia de la vacuna contra la influenza en pacientes oncológicos y con la información de éstos realizar estudios económicos para determinar la costo-efectividad de esta intervención en este grupo de pacientes. Dado que vacunar al personal de salud puede resultar en la disminución en el riesgo de adquirir la enfermedad en pacientes oncológicos esta práctica debería ser recomendada.

Introducir un programa para vacunar al personal de salud que tiene contacto estrecho con los pacientes oncológicos hospitalizados evita 10632 días de hospitalización y por lo tanto ahorra recursos por \$31 663 millones de pesos (US \$14 072 millones). El programa es costo-efectivo. Es además una estrategia dominante porque genera ahorros por cada unidad de resultado alcanzada, y continúa 
siendo dominante para una probabilidad de contagio mayor o igual a 0,02. Con una probabilidad de 0,01 la disminución de un día de estancia hospitalaria costaría \$3 248000 (US \$1 443) •

\section{REFERENCIAS}

1. Cooksley CD, Avritscher EB, Bekele BN, Rolston KV, Geraci JM, Elting LS. Epidemiology and outcomes of serious influenza-related infections in the cancer population. Cancer 2005; 104(3):618-28

2. Instituto Nacional de Salud. Epidemia de influenza A/H3N2 en Colombia. Informe Quincenal Epidemiológico Nacional 1996; 1(1):2-6.

3. Instituto Nacional de Salud. Epidemia de influenza A/H3N2/Beijing/32/92 en Ambalema Tolima 1994. Informe Quincenal Epidemiológico Nacional 1996; 1(1):6-8.

4. Instituto Nacional de Salud. Vigilancia epidemiológica para influenza y otros virus respiratorios. Informe Quincenal Epidemiológico Nacional 1998; 3(1):1-6

5. Instituto Nacional de Salud. Epidemia de influenza A/H3N2 en Colombia en 1996: actualización. Informe Quincenal Epidemiológico Nacional 1996; 1(3):39-41

6. Instituto Nacional de Salud. Influenza 1997: un nuevo aislamiento en Colombia. Informe Quincenal Epidemiológico Nacional 1997; 2(12): 172-173

7. Adult preventive health care: immunization [Internet]. Disponible en: http://www.guideline.gov. Consultado Julio de 2006.

8. Preventing pneumococcal disease among infants and young children [Internet]. Disponible en: http://www.guideline.gov. Consultado Julio de 2005.

9. Preventing pneumococcal disease among infants and young children. Norteamerica: Healthcare Infection Control Practices Advisory Committee [Internet]. Disponible en: http:// unw.guideline.gov. Consultado Julio de 2005.

10. Sumaya C, Williams T. Persistence of Antibody After the Administration of Influenza Vaccine to Children with Cancer. Pediatrics 1982; 69:226-229.

11. Brydak L, Calbecka M. Immunogenicity of Influenza Vaccine in Patients with Hemato Oncological Disorders. Leukemia and Lymphoma 1999; 32:369-374

12. Chisholm J, Devine T. Response to influenza immunization during treatment for cancer. Arch Dis Child 2001; 84:496-500

13. Wilde J, McMillan J, Serwint J; Butta J, O'Riordan MA; Steinhoff M. Effectiveness of Influenza Vaccine in Health Care Professionals: A Randomized Trial. JAMA 1999;281(10):908913.

14. Pearson M, Bridges C, Harper S. Influenza Vaccination of Health-Care Personnel. Recommendations of the Healthcare Infection Control Practices Advisory Committee (HICPAC) and the Advisory Committee on Immunization Practices (ACIP) [Internet]. Disponible en: www.cdc.gov/mmwr/preview/mmwrhtml/rr55e209a1.htm. Consultado Febrero 2006

15. Carman A, Elder L, Wallace K, McAulay A, Walker G, Murray D, et.al. Effects of influenza vaccination of health-care workers on mortality of elderly people in long-term care: a randomised controlled trial. The Lancet 2000;355(9198):93-97.

16. Potter J, Stott DJ, Roberts MA, Elder AG, O'Donnell B, Knight PV, et. al. Influenza vaccination of health care workers in long-term-care hospitals reduces the mortality of elderly patients. The Journal of Infectious Diseases 1997; 175(1):1-6.

17. Mesa S, Pérez A, Hurtado G, Arbeláez M. Effectiveness of an influenza vaccine in a working population in Colombia. Rev Panam Salud Publica 2001;10(4):232-239.

18. Salgado CD, Giannetta ET, Hayden FG, Farr BM. Preventing Nosocomial Influenza by Improving the Vaccine Acceptance Rate of Clinicians. Infection Control and Hospital Epidemiology 2004;25:923-928. 
766 REVISTADE SALUD PÚBLICA·Volumen 10 (5), Diciembre 2008

19. Avritscher EB, Cooksley CD, Geraci JM, Bekele BN, Cantor SB; Rolston KV, et.al. Costeffectiveness of influenza vaccination in working-age cancer patients. Cancer 2007 109(11):2357-64.

20. Fact Sheet for SARS Patients and Their Close Contacts [Internet]. Disponible en: http:// www.cdc.gov/ncidod/sars/factsheetcc.htm. Consultado Mayo 2006.

21. Instituto Nacional de Cancerología. Anuario Estadístico 2004. Bogotá: Instituto Nacional de Cancerología; 2005

22. Ruiz F, Amaya L, Garavito L, Ramirez J. Precios y contratos en salud estudio indicativo de precios y análisis cualitativo de contratos. Bogotá: Ministerio de la Protección Social; 2008.

23. Kempe A, Hall CB, MacDonald NE, Foye HR, Woodin KA, Cohen HJ, et al. Influenza in children with cancer. J Pediatr 1989;115(1):33-9.

24. Lin J, Zhang J, Dong X, Fang H, Chen J, Su N, et al. Safety and immunogenicity of an inactivated adjuvanted whole-virion influenza A (H5N1) vaccine: a phase I randomised controlled trial. Lancet 2006;368(9540):991-7.

25. Allison JE, Glezen WP, Taber LH, Paredes A, Webster RG. Reactogenicity and immunogenicity of bivalent influenza $A$ and monovalent influenza $B$ virus vaccines in high-risk children. Infect Dis 1977;136:S672-S676.

26. Treanor JJ, Campbell JD, Zangwill KM, Rowe T, Wolff M. Safety and immunogenicity of an inactivated subvirion influenza A (H5N1) vaccine. N Engl J Med 2006;354(13):1343-51. 